\title{
Understanding Mobile User Engagement with Pervasive Computing Systems
}

\author{
Mateusz Mikusz \\ School of Computing \& Communications \\ Lancaster University, Lancaster, UK \\ m.mikusz@lancaster.ac.uk
}

\section{MOTIVATION}

The vision of pervasive computing has always brought together mobile computing and fixed infrastructure systems. However, despite significant progress toward this vision, only a few tools are available to help developers and researchers understand the effectiveness of pervasive systems they create. My work aims to address this issue by providing support for pervasive computing analytics that will enable developers to obtain a deep understanding of how users interact with pervasive computing applications. The particular focus for my research is how mobile users interact with pervasive displays such as digital signage systems that have became a part of urban life and can be found at railway stations, airports and shopping malls. Although traditionally these displays are used for showing adverts and content such as timetables, researchers have started working on the next generation of digital signs that interact with mobile devices enabling viewer control and context-sensitive content-for example, adverts targeted at specific users $[4,15]$.

Current display analytics systems focus on reporting data for individual displays (such as view impressions, dwell time and demographics). However, cross-display tracking as users move through the physical environment and insights into viewer interactions across pervasive computing infrastructure and mobile phones are currently not available.

My work aims to address this shortcoming and offer analytics that focus on users rather than individual devices. The vision of this work is ultimately to help improve the quality and effectiveness of pervasive applications that run across mobile devices and digital signage. It will allow both developers and display providers to gain deeper insights into the interaction patterns of users. For example, analytics would be able to track users across multiple devices and pervasive displays and provide an "attraction measure" per application or display. This work will lay foundations for new forms of analytics that span across multiple devices and fixed infrastructure systems - and shift away from a device focused analytics view. While I am initially focusing on interactions between displays and mobile users I believe that many of the results will generalise to interactions with other pervasive computing components. This work was originally supported by a Google Faculty Award.

Permission to make digital or hard copies of part or all of this work for personal or classroom use is granted without fee provided that copies are not made or distributed for profit or commercial advantage and that copies bear this notice and the full citation on the first page. Copyrights for third-party components of this work must be honored For all other uses, contact the owner/author(s).

Ph.D. Forum'16 June 25-30 2016, Singapore, Singapore

(C) 2016 Copyright held by the owner/author(s).

ACM ISBN 978-1-4503-4331-2/16/06

DOI: http: //dx.doi .org/10.1145/2930056.2933328

\section{CHALLENGES}

New Metrics for Pervasive Interactions. State-of-the-art analytics systems such as Intel Anonymous Viewer Analytics [9] currently feature a very device-centric view of pervasive displays and, for example, provide audience demographics and impressions for specific displays. However, we believe that for the next generation of pervasive applications it will be essential to move toward analytics systems that focus around users instead of specific devices or displays. This will allow us to measure the effectiveness of such applications, user behaviour changes and interaction patterns across multiple mobile and pervasive devices. We will investigate how metrics and reports can be transformed to support such viewer-centric analytics. In addition to identifying the datasets, the ownership of the data needs to be considered. In a cross-device interaction chain multiple stakeholders may be involved (for example, viewers, application providers and pervasive display owners) with different sets of data gathered and owned by each stakeholder. To gain access to the entire information chain and combine multiple datasets owned by different stakeholders, both technical as well as legal requirements regarding data sharing attitudes need to be investigated.

Preserving User Privacy. Comprehensive tracking of user interactions across devices and locations is likely to be privacy invasive. For example, in order to measure the effectiveness of pervasive applications that work across mobile devices and multiple displays, analytics systems would need to be able to track both user interactions on the mobile phone and across displays. This level of tracking might lead to a record of location traces of individual users and allow the system to recognise the user across multiple domains. Therefore we believe the importance of obtaining relevant analytics data in a privacy preserving way is essential for the success and acceptance of such an analytics system.

\section{PROGRESS TO DATE}

My work is conducted in the context of the e-Campus infrastructure at Lancaster. This provides one of the world's largest research testbeds of pervasive displays consisting of approximately 50 public displays deployed in colleges, departmental buildings and lecture theatres. These displays are used to show a mix of local content (e.g. departmental news) and university-wide news. All displays are managed by e-Channels [6] and Mercury [3], and run Yarely [2].

My preparatory work consisted of extending this infrastructure in two distinct ways. Firstly, in order to support interactions with users and their mobile devices and to enable content scheduling decisions based on viewer preferences I designed and implemented a lottery-based content scheduler for the campus displays [10]. This scheduler supports multiple separate ticket allocation modules that 
allocate tickets to available content based on strategies such as giving recent content more tickets than other content. Secondly, in order to build an initial prototype of an analytics platform, I designed and deployed Pheme, an open platform that supports the collection of analytics data and the repurposing of existing third-party visualisation and analytics engines [11]. I use Pheme to collect relevant analytics data from our pervasive display deployment on campus and have further used it to explore application domains beyond displays such as IoT.

Building on these initial pieces of work I have now begun to explore how to provide user-centric analytics in a privacy preserving way. As deep insights into interaction patterns across devices and displays would require very detailed information about complex user behaviour patterns around and across displays, mobile phones and applications (with corresponding privacy concerns), I have instead designed a simulation-driven analytics framework that combines both simulated models of user behaviour with real-world analytics data. More specifically, the simulation framework is capable of generating synthetic user mobility traces on top of a spatial graph structure, and then uses these traces in combination with a variety of external data sources such as display content logs. This work builds on top of research that was conducted in the field of human mobility modelling (e.g. [7, 8]). Such models have been used for the simulation of routing in mobile ad-hoc networks [1, 5], evacuation of crowds $[13,16]$ or travelling patterns in the urban space [14]. For my application domain, my initial results show that the combination of synthetic traces of mobile users with real pervasive analytics datasets enables me to approximate interactions of users across multiple devices and generate a comprehensive set of reports such as detailed insights into mobility traces of the same user across multiple, distinctly located pervasive displays [12].

\section{CLOSING REMARKS}

To date I have laid the foundations for my work on pervasive analytics and begun to explore how a hybrid approach involving a mix of physical analytics data and simulation of user mobility can be used create new forms of analytics reports. In future work I hope to explore mobility models in more detail and gather real mobility traces (e.g. through WiFi fingerprints and mobile phone tracking) for both validating and improving existing models. From the $\mathrm{PhD}$ Forum I hope to gain feedback and engage in interesting discussions on the use of a simulation framework for user interactions with pervasive computing and, in particular, across mobile devices and pervasive displays.

\section{ACKNOWLEDGMENTS}

This research is partially funded through the Future and Emerging Technologies (FET) programme within the 7th Framework Programme for Research of the European Commission, under FET grant number: 612933 (RECALL) and was made possible with the support of a Google Faculty Research Award and Google Cloud Credits.

\section{REFERENCES}

[1] Aschenbruck, N., Frank, M., Martini, P., And TÖLLE, J. Human mobility in manet disaster area simulation-a realistic approach. In Local Computer
Networks, 2004. 29th Annual IEEE International Conference on (2004), IEEE, pp. 668-675.

[2] Clinch, S., Davies, N., Friday, A., And Clinch, G. Yarely - a software player for open pervasive display networks. In Proceedings of PerDis '13 (2013), ACM.

[3] Clinch, S., Mikusz, M., Greis, M., Davies, N., AND FRIDAY, A. Mercury: An application store for open display networks. In Proceedings of UbiComp '14 (2014), pp. 511-522.

[4] Farinella, G. M., Farioli, G., Battiato, S., LEONARDi, S., AND GALlo, G. Workshop for Video Analytics for Audience Measurement 2014. Springer, 2014, ch. Face Re-Identification for Digital Signage Applications, pp. 40-52.

[5] Feeley, M., Hutchinson, N., And Ray, S. Realistic mobility for mobile ad hoc network simulation. In Ad-Hoc, Mobile, and Wireless Networks. Springer, 2004, pp. 324-329.

[6] Friday, A., Davies, N., And Efstratiou, C. Reflections on long-term experiments with public displays. Computer, IEEE 45, 5 (May 2012), 34-41.

[7] Gonzalez, M. C., Hidalgo, C. A., and Barabasi, A.-L. Understanding individual human mobility patterns. Nature 453, 7196 (2008), 779-782.

[8] Hui, P., Chaintreau, A., Scott, J., Gass, R., Crowcroft, J., AND Diot, C. Pocket switched networks and human mobility in conference environments. In Proceedings of the 2005 ACM SIGCOMM workshop on Delay-tolerant networking (2005), ACM, pp. 244-251.

[9] INTEL CORP. Intel AIM Suite. https://aimsuite.intel.com/.

[10] Mikusz, M., Clinch, S., And Davies, N. Are you feeling lucky?: Lottery-based scheduling for public displays. In Proceedings of the 4th International Symposium on Pervasive Displays (New York, NY, USA, 2015), PerDis '15, ACM, pp. 123-129.

[11] Mikusz, M., Clinch, S., Jones, R., Harding, M., Winstanley, C., AND DaVies, N. Repurposing web analytics to support the iot. Computer 48, 9 (Sept 2015), 42-49.

[12] Mikusz, M., Noulas, A., Davies, N., Clinch, S., AND FRIDAY, A. Next generation physical analytics for digital signage. In Proceedings of the 3rd International Workshop on Physical Analytics (WPA) (2016).

[13] Pan, X., Han, C. S., Dauber, K., And Law, K. H. A multi-agent based framework for the simulation of human and social behaviors during emergency evacuations. Ai \& Society 22, 2 (2007), 113-132.

[14] Simini, F., González, M. C., Maritan, A., And BARABÁSI, A.-L. A universal model for mobility and migration patterns. Nature 484, 7392 (2012), 96-100.

[15] Tian, P., Sanjay, A. V., Chiranjeevi, K., And Malik, S. M. Intelligent advertising framework for digital signage. In Proceedings of the 18th ACM SIGKDD International Conference on Knowledge Discovery and Data Mining (New York, NY, USA, 2012), KDD '12, ACM, pp. 1532-1535.

[16] Zhong, M., Shi, C., Tu, X., Fu, T., AND He, L. Study of the human evacuation simulation of metro fire safety analysis in china. Journal of Loss Prevention in the Process Industries 21, 3 (2008), 287-298. 